\title{
Visible spectroscopy of extremely young asteroid families
}

\author{
T. Mothé-Diniz ${ }^{1, \star}$ and D. Nesvorný ${ }^{2, \star \star}$ \\ 1 Observatório Nacional, Rua Gal. José Cristino, 77, 20921-400 Rio de Janeiro, Brazil \\ e-mail: thais.mothe@on.br; thais.mothe@gmail.com \\ 2 Southwest Research Institute, 1050 Walnut St., Suite 300 Boulder, Colorado 80302, USA \\ e-mail: davidn@boulder.swri.edu
}

Received 9 April 2008 / Accepted 31 May 2008

\section{ABSTRACT}

\begin{abstract}
Context. Remotely identifying asteroid analogs of ordinary chondrites (OC) is difficult because the reflectance spectra of airless bodies can be modified by space weathering processes like micrometeorite impacts. Recently discovered extremely young asteroid families (ages $\leq 1 \mathrm{My}$ ) may not have suffered strong space weathering effects.

Aims. We study extremely young asteroid families to learn about the composition of main belt asteroids and the space weathering rate Methods. Members of extremely young families were, for the first time, observed and taxonomically classified. Observations took place at the GEMINI telescopes, using the instrument GMOS.

Results. The taxonomic classes found in the families are Q, Sq and S. The Q-class is the best proxy for OC materials.

Conclusions. We discovered for the first time Q-type asteroids in the Main-belt. Also,asteroids with $\leq 1 \mathrm{My}$ appear to have been only partially space weathered. These families are, therefore, very interesting targets for further observations in the near-infrared region.
\end{abstract}

Key words. minor planets, asteroids

\section{Introduction}

Meteorite specimens are remnants of the geologic processes that occurred in our Solar System. Properly analyzed, the bulk composition, mineralogy, and petrology of meteorites can be used to constrain planet formation processes. One fundamental difficulty with interpreting the meteorite record, however, is that we do not know (with a few exceptions) the location of their parent planetary bodies (or their immediate precursors). An evident case is the ordinary chondrite (OC) meteorites, which are the most common meteorites with the relative fall frequency of $75 \%$ (e.g. Burbine \& Binzel 2002), to which a few asteroids have been associated until now. This lack of main-belt objects spectrophotometrically similar to the OC meteorites is a long-debated and fundamental problem in planetary science.

It is now generally accepted that space weathering processes similar to those acting on the Moon (e.g. Pieters et al. 2000; Hapke 2001) can change optical properties of surfaces of airless bodies over time ${ }^{1}$. Many S-types ${ }^{2}$ may be ordinary chondritic in composition, but the diagnostic spectral shape and depth of absorption bands are likely space weathered with respect to those typical of OCs into a typical S-type spectrum, characterized

\footnotetext{
* Author T. Mothé-Diniz was supported by the Conselho Nacional de Desenvolvimento Científico e Tecnológico-CNPq/Brasil.

$\star \star$ The work of D.N. was supported by the NASA's Planetary Geology and Geophysics program.

1 Space weathering is the phenomenon, possibly due to micrometeorite impact and solar wind impingement, which modifies spectral reflectances from that of the inherent mineral assemblages.

${ }^{2}$ See, for example, Bus \& Binzel (2002) for definition of taxonomic types, including S-types.
}

by a reddish continuum and shallower absorption bands (Chapman 2004). Binzel et al. (1996) demonstrated a large variability of spectra of Near Earth Asteroids (NEAs), ranging from OC to traditional S-type, and suggested that they correspond to range of collisional lifetimes. Specifically, the recently "freshened" surfaces are similar to unmodified OC meteorites (from laboratory spectra) while progressively older asteroids are more maturely space weathered (reddened slope, shallower 0.9 and 2.0 micron bands). Published estimates of space weathering timescales range widely from $50 \mathrm{Ky}$ to $100 \mathrm{My}$ (Hapke 2001; Sasaki et al. 2001). Therefore, spectroscopic observations of very young families may be interesting for understanding how space weathering processes are affecting asteroids.

\section{Extremely young families}

The fundamental process affecting asteroids is their mutual catastrophic collisions. Collisions (a) create asteroid families (clusters of asteroid fragments with similar orbital elements); (b) produce the fragmental size distribution; (c) rearrange large "rubble pile" assemblages; and (d) provide delta-v's that help moving fragments into chaotic zones. Observations of asteroid families critically constrain asteroid collisional/dynamical evolution (Arnold 1969; Gradie et al. 1979; Chapman et al. 1989; Bottke et al. 2002; Zappalà et al. 2002).

A decade ago (e.g. Zappalà et al. 1990, 1994, 1995), we realized that (a) families are not very numerous, (b) most families are very old, hundreds of millions to billions of years, and (c) families generally represent the break-up of homogeneous precursor bodies (spectral properties of family members are usually similar and often distinct from background asteroids 
(Cellino et al. 2002). As asteroid discoveries rapidly increase, family identifications have also multiplied. About 50 asteroid families have been identified by Mothé-Diniz et al. (2005). To identify clusters of asteroids, Mothé-Diniz et al. (2005) searched for clusters of asteroid positions in the 3-dimensional space of the so-called proper orbital elements (Milani \& Knežević 1994; Knežević et al. 2002). These elements, which average over the rapidly varying osculating elements are proper semi-major axis $a_{\mathrm{P}}$, eccentricity $e_{\mathrm{P}}$, and inclination $i_{\mathrm{P}}$. Proper elements are dynamical quantities close to integrals of the motion of the system, which change very little over long time-scales, and therefore a cluster in proper-element space suggests common ancestry. It has been established that members of families gradually drift apart in space by radiation effects, like Yarkovsky (Bottke et al. 2002). Therefore, recently formed asteroid families are expected to be compact in $\left(a_{\mathrm{P}}, e_{\mathrm{P}}, i_{\mathrm{P}}\right)$ space.

Nesvorný et al. (2006b) and Nesvorný \& Vokrouhlický (2006) searched for compact families with just 1/100th of the dispersions in $\left(a_{\mathrm{P}}, e_{\mathrm{P}}, i_{\mathrm{P}}\right)$ seen in most known families. They identified four extremely young asteroid families by using an approach that differs from the traditional family-identification methods. A good description and comparison of such methods can be found in Bendjoya \& Zappalá (2002). Instead of using a catalog of proper orbital elements they used directly the osculating orbital elements. Because the osculating elements are not constant over My time intervals, they did not expect to find asteroid families older than $1 \mathrm{My}$. But extremely young families could show up as clusters in a 5-dimensional space of osculating orbital elements (not including mean anomaly which becomes dispersed by Keplerian shear within 10-1000 yr). Examining orbits of 264403 asteroids, they identified four new, extremely young asteroid clusters. Named after the largest members, these are Datura, Emilkowalski, 1992YC2, and Lucascavin clusters. To determine the formation ages, the orbits of cluster members were propagated backward in time. Given the small size of some of these asteroids (most members are $1-3-\mathrm{km}$ in diameter), the computer simulations accounted for radiation forces like the Yarkovsky Effect. The results showed that Datura, Emilkowalski, 1992YC2, and Lucascavin clusters are $450+/-50$ thousand years (ky), 220+/-30 ky, 50-250 ky, and 200-600 ky old, respectively. They are the most recent asteroid breakups ever discovered in the main belt.

Previously, Nesvorný et al. (2002, 2003) had determined ages of three slightly older families: the Karin cluster $(5.75+/-0.05 \mathrm{My})$, Veritas family $(8.3+/-0.3 \mathrm{My})$, and Iannini family $(<5 \mathrm{My})$. The spectroscopic observations in 2004-6 showed that Karin and Iannini cluster members are S-complex asteroids. These families have been studied by a number of observers (Yoshida et al. 2004; Sasaki et al. 2004, 2005; Hahn et al. 2006; Chapman et al. 2007; Vernazza et al. 2006). The observations showed that (832) Karin, well-sampled throughout its rotation, exhibits no significant color variation with rotation. It appears that the members of the Karin family are not quite as red as typical S-types, yet the absorption bands are quite shallow. A possible explanation is that the space-weathering process affecting these young family members has had time to reduce the spectral contrast of the reflectance spectra, but has not operated long enough to redden them - an intermediate case of space weathering, which has gone to completion for older asteroids of these sizes in the main belt (Chapman et al. 2007). Preliminary reflectance spectra for members of the Iannini cluster members appear to be similar to those of Karin (Willman et al. 2008).

The observations of Datura, Emilkowalski, 1992YC2, and Lucascavin clusters that we performed here have very important implications for the trends discussed above. As these families are $\simeq 10$ times younger than the Karin cluster, it would be expected that the space weathering effects should be significantly less pronounced for them. We describe our observations in Sect. 3 and discuss the results in Sect. 4.

\section{Observations}

Observations were performed with the two Gemini Multi-Object Spectrographs (GMOS) (Hook et al. 2004), one at each GEMINI telescope. Observations in the first semester of 2007 were made in classical mode, while the programs in the second semester of 2007 were made in queue mode. Both cameras have a pixel size of $0.0727^{\prime \prime} /$ pixel with a field of view of $330 \times 330 \operatorname{arcsec}^{2}$. The spectrographs were used in the long-slit mode, with a slit of $1.5^{\prime \prime}$. The filter GG455-G0305 was used for the observations, making the wavelength interval $\geq 460 \mathrm{~nm}$ and the effective wavelength $\geq 550 \mathrm{~nm}$. This filter was used with the grating R150+G5306 for Gemini North and the identical grating R150+G5326 for Gemini South, which yields a simultaneous coverage of $1071 \mathrm{~nm}$, with an $R=631$ and the Blaze wavelength is at $717 \mu \mathrm{m}$. Both GMOS-N and GMOS-S have significant fringing in the red (from $0.7 \mu \mathrm{m}$ on). To minimize these effects, for every science object we obtained an even number of spectra, pairs of spectra in two positions of the slit, and performed subtractions B-A. Calibration images of Flat and Arc were obtained via the Gemini Facility Calibration Unit (GCAL), with the same configuration as the science data. After the whole sequence of every object was concluded, three flat-field images and one arc were taken for calibration. The lamp available for arc calibration of optical spectra is the CuAr.

We obtained good S/N data for asteroids (1270), (16598), 2001 WY35, and 2004 XL40. The useful spectral range is 0.4-0.92 $\mu \mathrm{m}$. Objects (89309), 2000UV80, and (1 226761) were also observed in these programs, but either because of the quality of the spectra or because of problems during observing in service mode, they could not be used.

\subsection{Reduction}

The reduction of the data was made using the standard Gemini IRAF package, with the sub-package GMOS. Biases were combined with the task "gbias", flats were combined using the task "gsflat", and science targets were reduced with task "gsreduce", which subtracts off the bias, mosaics the three detectors of GMOS, interpolates across the chip gaps for science data, and applies flat field correction. Then the $\mathrm{CuAr}$ images were also reduced with "gsreduce". For these arcs, the wavelength calibration was established with the task "gswavelength", and the science spectra were further transformed by applying the task "gstransform". Then the sky subtraction was done with "gsskysub", and the pairs of spectra A and B were subtracted, so that we obtained one positive and one negative spectrum. We then extracted all the negative and the positive spectra for every target. The same process was used for the solar analog stars, with the exception of the subtraction A-B. For the division of the asteroid spectra by the solar analogs, all spectra of every science target and solar analog were summed up. The final spectrum of each asteroid was then and re-binned to match the same points of the star. The spectra were then rationed by the solar analog spectrum and normalized to unity at $0.55 \mu \mathrm{m}$. In Table 1 we present the details of the observation of the targets, like UT date, exposure times, phase angle, apparent magnitude, and the solar analog used for removing the solar flux from the asteroid. 
Table 1. Observational details for asteroid spectra of the members of the young families presented in this paper.

\begin{tabular}{lcccccl}
\hline \hline Asteroid & UT date & $\begin{array}{c}\text { Exposure } \\
(\mathrm{s})\end{array}$ & $\begin{array}{c}\text { Mean } \\
\text { airmass }\end{array}$ & $\begin{array}{c}\text { Phase angle } \\
(\mathrm{deg})\end{array}$ & Mag. (V) & $\begin{array}{l}\text { Solar } \\
\text { Analog }\end{array}$ \\
\hline (1270) Datura & 16-Nov.-2007 & $4 \times 250$ & 1.17 & 24.9 & 16.7 & SA98682 \\
(60 151) 1999 UZ6 & 15-Mar.-2007 & $4 \times 360$ & 1.80 & 19.8 & 20.5 & SA144335 \\
(90 265) 2003 CL5 & 15-Mar.-2007 & $2 \times 250+8 \times 300$ & 1.20 & 8.2 & 19.3 & SA144335 \\
2001 WY35 & 2-Sep.-2007 & $4 \times 300$ & 1.09 & 29.9 & 21.5 & SA1112093 \\
(16 598)1992 YC2 & 17-Aug.-2007 & $4 \times 300$ & 1.28 & 17.2 & 20.3 & SA1112093 \\
2004 XL40 & 20-Aug.-2007 & $4 \times 280$ & 1.28 & 15.6 & 20.4 & SA1112093 \\
\hline
\end{tabular}

Table 2. Physical data, taxonomic classification of the asteroids, and estimated ages for the breakup of their corresponding families.

\begin{tabular}{lcccccccc}
\hline \hline Asteroid & $\begin{array}{c}H \\
(\mathrm{mag})\end{array}$ & $\begin{array}{c}D \\
(\mathrm{~km})\end{array}$ & $\begin{array}{c}a_{\mathrm{P}} \\
(\mathrm{AU})\end{array}$ & $e_{\mathrm{P}}$ & $i_{\mathrm{P}}$ & $\begin{array}{c}\text { Taxon. } \\
\text { class }\end{array}$ & Family & $\begin{array}{c}\text { Age of } \\
\text { family }^{a}\end{array}$ \\
\hline (1270) Datura & 12.5 & 10.8 & 2.23 & 0.15 & 5.33 & $\mathrm{Sk}$ & Datura & $450 \pm 50 \mathrm{Ky}$ \\
(60 151) 1999 UZ6 & 16.3 & 1.9 & 2.23 & 0.15 & 5.32 & $\mathrm{Sk}$ & Datura & $450 \pm 50 \mathrm{Ky}$ \\
(90 265) 2003 CL5 & 15.4 & 2.9 & 2.23 & 0.15 & 5.32 & $\mathrm{Sq}$ & Datura & $450 \pm 50 \mathrm{Ky}$ \\
2001 WY35 & 17.0 & 1.4 & 2.23 & 0.15 & 5.33 & $\mathrm{O} / \mathrm{Q}$ & Datura & $450 \pm 50 \mathrm{Ky}$ \\
(16 598)1992 YC2 & 14.7 & 3.9 & 2.59 & 0.18 & 17.22 & $\mathrm{Sq}$ & $(16598)$ & $50-250 \mathrm{Ky}$ \\
2004 XL40 & 17.0 & 1.4 & 2.28 & 0.13 & 5.31 & $\mathrm{Sq}$ & Lucascavin & $300-800 \mathrm{Ky}$ \\
\hline
\end{tabular}

${ }^{a}$ Determined by Nesvorný et al. (2006a), and the effective diameters $(D)$ were estimated assuming an albedo $p_{v}=0.15$.
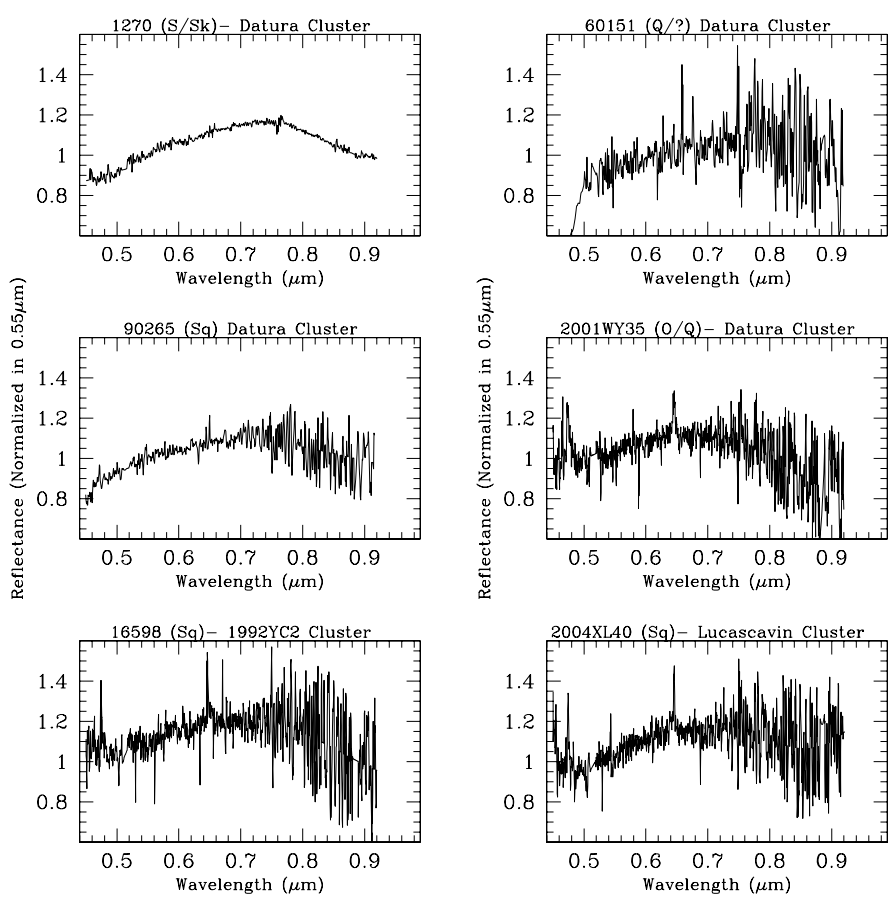

Fig. 1. Spectra of the objects observed in this study, normalized to unity at $0.55 \mu \mathrm{m}$.

\section{Results}

Figures 1 and 2 show individual plots of each obtained spectrum and of the resulting spline fits of all the spectra, respectively. The visible spectra of all the objects belonging to extremely young families observed exhibit slightly reddish spectral slope and pronounced absorption band near $0.9 \mu \mathrm{m}$. For classifying the spectra we used our own implementation of the algorithm developed and described by Bus \& Binzel (2002), in which an asteroid belongs to a given taxonomic class according to the presence or absence of specific features in its visible spectrum (from 0.44 to $0.92 \mu \mathrm{m}$ ) and to values of particular parameterized spectral characteristics. The spectra presented in this paper belong to the subclasses Sq,

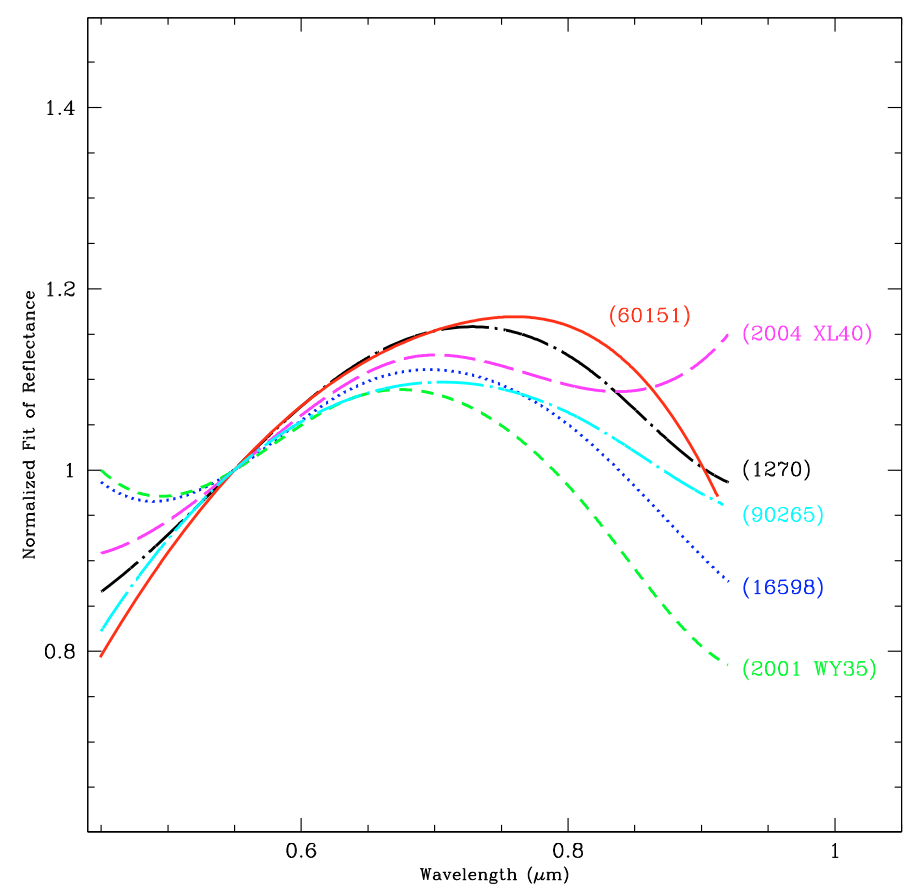

Fig. 2. Spline fit of the objects observed. All fits were normalized to unity at $0.55 \mu \mathrm{m}$.

Sk, Q, and O of the S-complex of Bus. The precise definition of each of the Bus complexes, and subclasses is detailed in Bus \& Binzel (2002), but in general lines, the spectra belonging to the $\mathrm{S}$-complex all have the common characteristic of a moderate to steep red slope from 0.44 to $0.70 \mu \mathrm{m}$, usually reaching a maximum reflectance between 0.72 and $0.76 \mu \mathrm{m}$. Longward of this peak in the reflectance, the spectra range from being flat to having a very deep silicate band whose minimum reflectance band is around $1.0 \mu \mathrm{m}$. The subclasses $\mathrm{O} / \mathrm{Q}$ and $\mathrm{Sq}$ of the $\mathrm{S}$-complex found among the young families observed all have as a common characteristic a relatively strong $1.0 \mu \mathrm{m}$ band and steep UV slope. It is worth noticing that in the principal components space there is some intersection between Sk and Sq classes. 


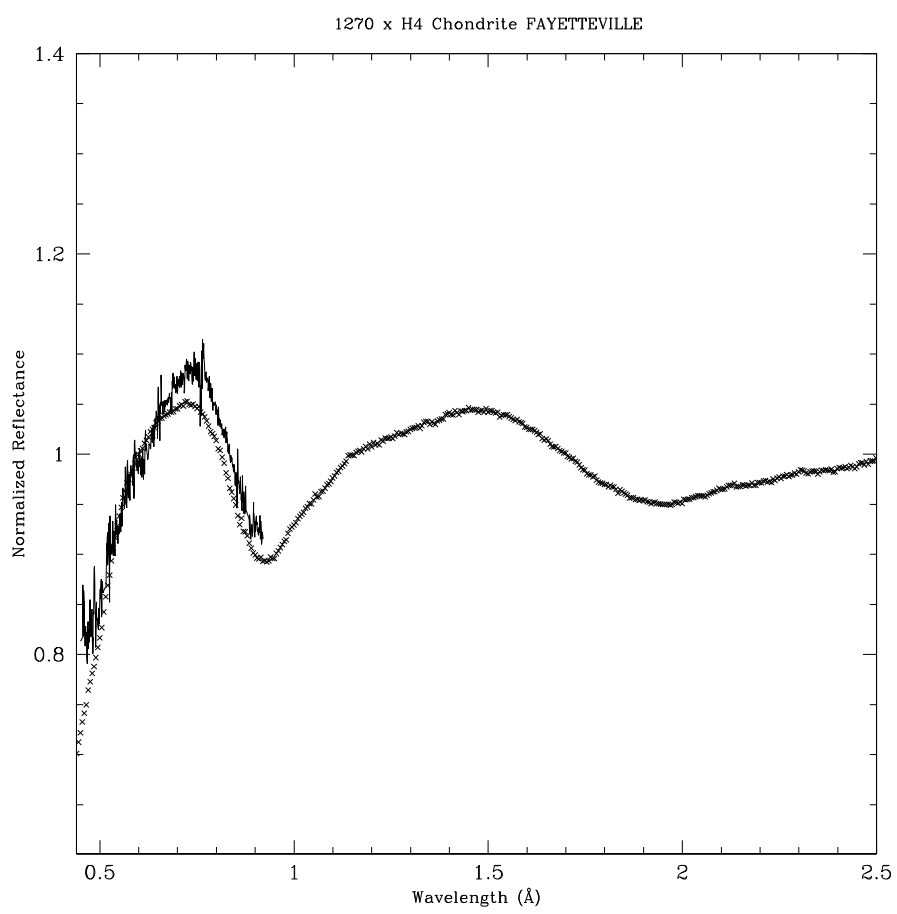

Fig. 3. Spectrum of (1270) Datura plotted with the spectrum of the ordinary chondrite Fayetteville.

The detailed classification of the spectra for individual objects is listed in Table 2. In our group of observations, only objects (1270) and (60151) from the Datura family have slightly flatter spectral slopes and smaller $1.0 \mu \mathrm{m}$ band depths, belonging to the taxonomic class Sk. The other four objects belong to taxonomic classes $\mathrm{Sq}, \mathrm{Q}$, and $\mathrm{O}$. We compared these spectra with laboratory spectra of OCs. Figure 3 shows the comparison of the spectrum of object (1270) Datura with the spectrum of an $\mathrm{H} 4$ ordinary chondrite. Both spectra are very similar in the visible range, with the $\mathrm{OC}$ one presenting just a slightly smaller reflectance in a region around $0.7 \mu \mathrm{m}$. Observations in the nearinfrared would be needed to establish whether the spectral similarities persist in the wavelength range $0.9-2.5 \mu \mathrm{m}$.

\section{Conclusions}

In this work we obtained visible spectra of asteroids formed by recent break-ups (Nesvorný et al. 2006b). From the spectral characteristics observed, our results strongly suggest that all the young families defined by Nesvorný et al. (2006b) may have suffered just minimal space weathering. As these families are younger than $1 \mathrm{Myr}$, this result agrees with the estimated timescales between $50 \mathrm{Ky}$ to $100 \mathrm{My}$ for the space weathering to act on silicate surfaces (Hapke 2001; Sasaki et al. 2001). Four of the objects observed in this study belong to the taxonomic classes Sq or Q, presenting a low visible spectral slope and suggesting deep $1.0 \mu \mathrm{m}$ band, which are the characteristics of the visible spectra of OCs. Two other objects belong to the class Sk, which presents intersections to the class $\mathrm{Sq}$ in the principal components space. The main result of this work is that we have discovered for the first time Q-type asteroids in the main-belt. These Q-types are among very small objects, belonging to the youngest asteroid families found.

Acknowledgements. This work is based on observations obtained at the Gemini Observatory, which is operated by the Association of Universities for Research in Astronomy, Inc., under a cooperative agreement with the NSF on behalf of the Gemini partnership: the National Science Foundation (United States), the Science and Technology Facilities Council (UK), the National Research Council (Canada), CONICYT (Chile), the Australian Research Council (Australia), CNPq (Brazil), and SECYT (Argentina) We are grateful to all the staff of GEMINI telescopes who assisted us in the observational programs and especially to Rodrigo Carrasco and Susan Ridgway, who were in a direct contact with us during the whole preparation of Phase II and the observations in the classical mode turn, with patience, and professionalism. We thank also the reviewer, Alberto Cellino, for his helpful comments that helped make this paper more complete.

\section{References}

Arnold, J. R. 1969, AJ, 74, 1235

Bendjoya, P., \& Zappalá, V. 2002, Asteroids III, 613

Binzel, R. P., Bus, S. J., Burbine, T. H., \& Sunshine, J. M. 1996, Science, 273, 946

Bottke, W. F., Morbidelli, A., Jedicke, R., et al. 2002, Icarus, 156, 399

Burbine, T. H., \& Binzel, R. P. 2002, Icarus, 159, 468

Bus, S. J., \& Binzel, R. P. 2002, Icarus, 158, 146

Cellino, A., Bus, S. J., Doressoundiram, A., \& Lazzaro, D. 2002, Asteroids III, 633

Chapman, C. R. 2004, Ann. Rev. Earth Plan. Sci., 32, 539

Chapman, C. R., Paolicchi, P., Zappala, V., Binzel, R. P., \& Bell, J. F. 1989, in Asteroids II, ed. R. P. Binzel, T. Gehrels, \& M. S. Matthews, 386

Chapman, C. R., Enke, B., Merline, W. J., et al. 2007, Icarus, 191, 323

Gradie, J. C., Chapman, C. R., \& Williams, J. G. 1979, Families of minor planets, Asteroids, 359

Hahn, G., Mottola, S., Sen, A. K., Harris, A. W., \& Mueller, E. K. M. 2006, Bull. Astron. Soc. India, 34, 393

Hapke, B. 2001, J. Geophys. Res., 106, 10039

Hook, I. M., Jørgensen, I., Allington-Smith, J. R., et al. 2004, PASP, 116, 425

Knežević, Z., Lemaître, A., \& Milani, A. 2002, Asteroids III, 603

Milani, A., \& Knežević, Z. 1994, Icarus, 107, 219

Mothé-Diniz, T., Roig, F., \& Carvano, J. M. 2005, Icarus, 174, 54

Nesvorný, D., \& Vokrouhlický, D. 2006, AJ, 132, 1950

Nesvorný, D., Bottke, Jr., W. F., Dones, L., \& Levison, H. F. 2002, Nature, 417, 720

Nesvorný, D., Bottke, W. F., Levison, H. F., \& Dones, L. 2003, ApJ, 591, 486

Nesvorný, D., Vokrouhlicky, D., \& Bottke, W. F. 2006a, Science, 312, 1490

Nesvorný, D., Enke, B. L., Bottke, W. F., et al. 2006b, Icarus, 183, 296

Pieters, C. M., Taylor, L. A., Noble, S. K., et al. 2000, Meteoritics and Planetary Science, 35, 1101

Sasaki, S., Nakamura, K., Hamabe, Y., Kurahashi, E., \& Hiroi, T. 2001, Nature, 410,555

Sasaki, S., Ueda, Y., Loeffler, M., \& Hiroi, T. 2004, in COSPAR, Plenary Meeting, 35th COSPAR Scientific Assembly, 35, 3732

Sasaki, T., Sasaki, S., Watanabe, J. I., et al. 2005, in Lunar and Planetary Institute Conference Abstracts, 36th Annual Lunar and Planetary Science Conference, ed. S. Mackwell, \& E. Stansbery, 36, 1590

Vernazza, P., Birlan, M., Rossi, A., et al. 2006, A\&A, 460, 945

Willman, M., Jedicke, R., Nesvorný, D., et al. 2008 [arXiv: 0802 .2977v1]

Yoshida, F., Dermawan, B., Ito, T., et al. 2004, PASJ, 56, 1105

Zappalà, V., Cellino, A., Farinella, P., \& Knežević, Z. 1990, AJ, 100, 2030

Zappalà, V., Cellino, A., Farinella, P., \& Milani, A. 1994, AJ, 107, 772

Zappalà, V., Bendjoya, P., Cellino, A., Farinella, P., \& Froeschlé, C. 1995, Icarus, 116,291

Zappalà, V., Cellino, A., Dell'Oro, A., \& Paolicchi, P. 2002, Asteroids III, 619 